\title{
Carcinoma Não Medular Familiar da Tiróide
}

\section{RESUMO}

O carcinoma diferenciado da tiróide, papilífero ou folicular, origina-se da célula folicular tiroideana, sendo a neoplasia maligna mais freqüente desta glândula. Desde 1955 têm sido relatados casos de agrupamento familiar deste carcinoma, e atualmente estima-se que $4,2 \%$ de todos os carcinomas diferenciados da tiróide tenham origem familiar. Esses casos costumam ser mais agressivos, incidem em idade mais precoce, são multifocais e apresentam maior taxa de recorrência. Parecem ser transmitidos por herança autossômica dominante com penetrância variável, mas os genes exatos responsáveis pela doença ainda não foram totalmente identificados. Os pacientes devem ser tratados com tiroidectomia total e freqüentemente também com esvaziamento linfonodal cervical, seguidos de ablação com iodo radioativo e terapia supressiva do TSH com levotiroxina. Alguns autores recomendam rastreamento de familiares de primeiro grau dos pacientes afetados através da ultrassonografia cervical, com objetivo de realizar diagnóstico precoce, possibilitando melhores resultados terapêuticos.

\section{(Arq Bras Endocrinol Metab 2007;51/5:769-773)}

Descritores: Tiróide; Câncer da tiróide; Marcadores moleculares; Terapêutica

\section{ABSTRACT}

Familial Nommedullary Thyroid Cancer.

Nonmedullary thyroid carcinoma, originating from thyroid epithelial cells, is the most frequent thyroid malignant neoplasia. Since 1955, there has been increasing evidence that this cancer may have a familial predisposition. It is now established that around $4.2 \%$ of all nonmedullary thyroid carcinomas occurs on the background of familial predisposition. These cases are often more aggressive, due to early onset, multifocality and a higher percentual of recurrences. An autossomal dominant inheritance pattern appears likely in most families, although the exact genes responsible for this syndrome have not yet been identified. Patients affected by this cancer should be treated with total thyroidectomy routinely and, in most cases, lymph node dissection, followed by iodine ablation and TSH suppressive therapy with levothyroxine. Some authors also recommend that first-degree relatives of patients with nonmedullary thyroid cancer (especially women) should be submitted to neck ultrasound for thyroid cancer screening, aiming early diagnosis for better treatment results. (Arq Bras Endocrinol Metab 2007;51/5:769-773)

Keywords: Thyroid; Thyroid cancer; Molecular markers; Therapeutics

A GRANDE MAIORIA DAS NEOPLASIAS malignas tiroideanas tem origem na Lcélula folicular, sendo chamada de carcinomas diferenciados da tiróide (CDT). Histologicamente, podem ser papilíferos ou foliculares. Caracterizam-se por serem esporádicos, entretanto diversos trabalhos têm demonstrado um risco aumentado de 3,21 a 10,3 vezes de CDT em familiares de pacientes afetados por essa neoplasia (1-3). Em 1955, Robinson e Orr relataram casos de câncer da tiróide não medular em gêmeos monozigóti-

\section{revisão}

\section{ROBERTO BERNARDO SANTOS THAÍS GOMES DE MELO LÍGIa V. Montali AsSUMpÇÃo}

Serviço de Endocrinologia, Centro de Ciências da Vida, Pontifícia Universidade Católica de Campinas (RBS); Disciplina de Endocrinologia, Departamento de Clínica Médica, Universidade Estadual de Campinas (TGM, LVMA), Campinas, SP.
Recebido em 06/02/07 Aceito em 09/02/07 
cos. A partir dessa data, numerosos relatos de carcinoma da tiróide não medular familiar (CTNMF) foram publicados, sendo então reconhecido como uma entidade clínica distinta (4).

\section{DEFINIÇÃO}

O diagnóstico do CTNMF se baseia na presença de dois ou mais parentes de primeiro grau afetados pelo câncer da tiróide (papilífero, folicular ou de células de Hürthle). A associação de carcinoma diferenciado da tiróide com síndromes familiares como a doença de Cowden, polipose adenomatosa familiar, complexo de Carney ou a exposição a fatores ambientais conhecidos por predispor a essa neoplasia invalidam esse diagnóstico (5).

Estima-se que se dois membros da família são afetados por CDT há uma chance de $53 \%$ de este câncer ter uma origem familiar e $47 \%$ de ser esporádico. Se três ou mais membros da família são afetados, há $99 \%$ de probabilidade de esta doença ser familiar $(6,7)$.

A prevalência do CTNMF varia de $1,8 \%$ a $10,5 \%$, com uma média em 4,2\% dentre todos os pacientes com CDT (8-10).

\section{CARACTERÍSTICAS}

Além da história familiar, não há diferenças entre as características clínicas ou histológicas do carcinoma diferenciado da tiróide na sua forma esporádica e familiar. $\mathrm{O}$ tipo histológico mais comum é o papilífero, sendo o carcinoma de células de Hürthle e o carcinoma folicular os mais raros. $\mathrm{O}$ carcinoma papilífero familiar está associado a maior incidência de condições benignas tiroideanas (adenoma folicular, bócio multinodular e tiroidite) (11). Como na forma esporádica, as mulheres são 2 a 3 vezes mais afetadas que os homens, porém com início de doença numa faixa etária um pouco mais jovem.

Podem apresentar-se como multicêntricos, bilateralmente ou ambos em aproximadamente $40 \%$ a $50 \%$ dos casos. Extensão metastática precoce para linfonodos locorregionais e metástases a distância ocorrem em um considerável número de pacientes. Grossman encontrou, num grupo de 14 pacientes com CTNMF, multicentricidade em $93 \%$, bilateralidade em $43 \%$, invasão local associada a metástase linfonodal em $57 \%$. Metade dos pacientes apresentaram recorrência durante o seguimento (12).

Uchino, em trabalho retrospectivo, avaliou 258 pacientes com CTNMF, comparando-os a 6.200 pacientes com a forma esporádica. Observou que os pacientes com a forma familiar apresentavam maior disseminação intra-glandular (40,7\% x 28,5\%), maior associação com bócio multinodular benigno (41,5\% x $29,8 \%)$, recorrência de doença mais freqüente (16,5\% x 9,6\%) e tempo livre de doença mais curto (10).

Mesmo microcarcinomas apresentaram-se com um comportamento mais agressivo, com metástases pulmonares e óbito subseqüente (8). Outros trabalhos, entretanto, não observaram este tipo de agressividade. Em um estudo de caso controle realizado em um hospital de Toronto (Canadá), comparou-se 24 casos de CTNMF com 543 casos da forma esporádica, não encontrando diferenças com relação ao tempo livre de doença e, portanto, sem apresentar pior prognóstico (13).

Triponez, em estudo retrospectivo, avaliou a sobrevida de 139 pacientes com CTNMF, no período de 1974 a 2004. Observou que após tratamento, o carcinoma não afetou significativamente a sobrevida de pacientes quando comparados com membros de sua família não acometidos ou com a população geral dos Estados Unidos. Contudo, aqueles que faziam parte de famílias com três ou mais membros afetados apresentavam uma sobrevida mais curta quando comparados com famílias com somente dois membros acometidos (14).

\section{TRATAMENTO}

Recomenda-se tiroidectomia total para remover todo o tecido tiroideano devido à predisposição à multicentricidade, bilateralidade e pelo possível comportamento mais agressivo desses tumores. Além disso, permite que a tiroglobulina seja utilizada eficazmente como marcador tumoral e também a utilização do iodo radioativo para doses ablativas e pesquisa de metástases. Isto facilita a detecção de recorrência, persistência ou de metástases da doença. Se o tumor primário tem 10 milímetros ou mais, sugere-se que os pacientes sejam submetidos à dissecção profilática de linfonodos ipsilaterais ou centrais bilaterais no mesmo tempo cirúrgico (6).

Recomenda-se a administração de dose ablativa de radioiodo após a cirurgia mesmo em pacientes com baixo risco, pois reduz o risco de recorrência e facilita o seguimento com a tiroglobulina, sobretudo para pacientes com tumor primário maior que 10 milímetros ou com envolvimento linfonodal. A recorrência cervical deveria ser tratada por uma dissecção radical modificada do pescoço. A princípio, valores de TSH devem ser mantidos suprimidos, em torno de 0,1 $\mathrm{mUI} / \mathrm{L}$. Em pacientes de alto risco, o valor de TSH deve permanecer inferior a $0,1 \mathrm{mUI} / \mathrm{L}$ (15).

Para os familiares de primeiro grau de pacientes afetados pelo CTNMF, seria importante a 
realização de investigação tiroideana ultrassonográfica inicialmente e, se não encontrada alteração, numa freqüência regular a cada 1 ou 2 anos. Isso levaria a uma detecção mais precoce do tumor e, conseqüentemente, melhor evolução. $\mathrm{O}$ achado de nódulos com tamanho igual ou superior a l centímetro obriga a realização de punção aspirativa por agulha fina para diagnóstico citológico. Não há um consenso com relação à punção de nódulos com tamanho inferior a 1 centímetro, pela dificuldade em coletar amostras adequadas para análise. Se a opção, nesses casos, for de observação, deve haver um acompanhamento rigoroso quanto ao seu crescimento, com punção se atingir 1 centímetro de tamanho (16). Outros autores recomendam a realização de tiroidectomia para todo paciente com nódulo tiroideano com história fortemente sugestiva de CTNMF, independentemente do resultado da punção aspirativa. Alegam que a punção nesses pacientes apresentaria menor sensibilidade devido à incidência de carcinoma multifocal e a alta coexistência de nódulos benignos (17).

Uchino realizou investigação ultrassonográfica tiroideana em 149 membros de 53 famílias de CTNMF não acometidos até aquele momento. Seu objetivo foi investigar a incidência e as características da doença nesse grupo de risco. Um ou mais nódulos estavam presentes em $52 \%$ dos pacientes $(n=77)$, que foram avaliados através de punção aspirativa. Destes, 15 (1 homem e 14 mulheres) tiveram diagnóstico de carcinoma papilífero através de cirurgia. Recomenda que, pela alta incidência de neoplasia neste grupo estudado $(10,1 \%)$, todos os parentes de primeiro grau de pacientes com CTNMF (especialmente mulheres) devam realizar investigação ultrassonográfica da tiróide (18).

\section{DOENÇAS ASSOCIADAS}

Estudos populacionais e de metanálise realizados para avaliar as características do CTNMF mostraram maior freqüência de outras doenças tiroideanas e neoplasias nas famílias afetadas $(5,10,11,16)$. Tiroidite de Hashimoto, hipotiroidismo e hipertiroidismo também são mais freqüentes em famílias com CTNMF $(2,19)$. Os estudos sugerem, ainda, maior risco de desenvolvimento de neoplasias primárias de outros sítios, como carcinoma de mama, rim, cólon direito, ovário, melanoma, linfoma, neurinomas e tumores do tecido conjuntivo $(1,2,19)$. Somente uma análise populacional realizada na Noruega em 2001 não confirmou este risco $(20)$.

\section{ASSOCIAÇÕES SINDRÔMICAS COM CARCINOMÁ DIFERENCIADO DA TIRÓIDE}

As síndromes descritas abaixo estão associadas com maior risco de CDT, sendo que sua presença invalida o diagnóstico de CTNMF.

1. Polipose adenomatosa familiar (PAF): caracterizada pelo desenvolvimento de múltiplos pólipos adenomatosos na mucosa do trato gastrintestinal com potencial de transformação maligna. É causada por mutação germinativa inativadora do gene supressor tumoral APC (localizado no cromossomo 5q21) e transmitida por herança autossômica dominante. Um a dois por cento dos casos apresentam carcinoma papilífero da tiróide, principalmente de padrão histológico cribiforme (21).

2. Síndrome de Gardner: variante da PAF, caracterizada pela associação de pólipos e manifestações extra-colônicas, como dentes supra-numerários, displasia fibrosa do crânio, osteomas da mandíbula, fibromas, tumores desmóides, cistos epiteliais, hamartomas do trato gastrointestinal, hepatoblastomas. Tumores de tiróide ocorrem em $2 \%$ dos casos (5).

3. Doença de Cowden: caracterizada por hamartomas múltiplos e tumores de mama, cólon, endométrio e cérebro, é associada à mutação do gene supressor tumoral PTEN (cromossomo 10q22-23). A manifestação extra-cutânea mais comum é a doença tiroideana (bócio, tiroidites, neoplasias), que ocorre em dois terços dos casos, sendo de $9 \%$ a incidência de carcinoma folicular (22).

4. Doença de Werner: caracterizada por envelhecimento prematuro, alterações cutâneas esclerodérmicas, catarata, calcificações subcutâneas, atrofia muscular, diabetes mellitus e maior risco de neoplasias. Causada por mutação do gene WRN (cromossomo 8p11-21). Carcinomas da tiróide, especialmente folicular, ocorrem precocemente (5).

5. Complexo de Carney: associação de mixomas de partes moles, pigmentação cutânea e mucosa, schwannomas, tumores de adrenal, hipófise e testículos. Causada por mutação do gene PPKARl alfa (cromossomo $17 \mathrm{q} 24$ ). Tumores da tiróide, mais freqüentemente benignos, ocorrem em 10 a $25 \%$ dos pacientes (23).

\section{GENÉTICA DO CARCINOMA DA TIRÓIDE NĀO MEDULAR FAMILIAR}

A introdução da pesquisa de mutações do oncogene RET nos casos de carcinoma medular da tiróide trouxe avanços no diagnóstico e tratamento desta neoplasia 
(24). Do mesmo modo, a identificação dos genes responsáveis pelo CTNMF possivelmente facilitaria o rastreamento e o diagnóstico precoce da doença, aumentando as taxas de cura e de sobrevida.

Analisando as várias famílias acometidas pelo CTNMF relatadas na literatura, observa-se acometimento de vários membros na mesma geração e transmissão por genitores de ambos os sexos (25-31), o que sugere transmissão genética por padrão de herança autossômico dominante, com penetrância variável.

Osaki mostrou que o HLA B7 e o DRl são encontrados mais freqüentemente nos casos de CTNMF que nos CDT esporádicos (32). Em seis famílias com CTNMF estudadas por Orzenigo houve maior positividade para HLA B7, DR5 e CW7 (28), confirmando mais uma vez a base genética do carcinoma.

Há muito tempo se estudam as alterações genéticas do CDT esporádico e algumas já foram identificadas: rearranjos RET/PTC, mutações dos gene ras, TRK e BRAF, translocação PAX8-PPARg, dentre outras. Entretanto, nenhuma destas anormalidades foi detectada no CTNMF $(33,36)$.

Até o momento, já foram identificados através de linkage analysis três genes que possivelmente estão relacionados ao desenvolvimento do CTNMF: TCO, PRNl e NMTCl (5).

$\mathrm{O}$ gene TCO, localizado no cromossomo 19 pl3.2, foi identificado em uma família francesa com recorrência de tumores tiroideanos oxifílicos, por Canzian, em 1998 (34). Sua relação com CTNMF foi confirmada em estudo posterior realizado por Bevan (35). Porém, McKay, ao estudar uma grande família da Tasmânia com oito membros afetados por CDT, não demonstrou significância desse lócus (36).

O gene PRN1 localiza-se no cromossomo lq21 e foi mapeado numa família norte-americana acometida por carcinoma papilífero da tiróide, bócio nodular e neoplasia papilífera renal (37).

O gene NMTCl localiza-se no cromossomo 2q2l e foi detectado na grande família da Tasmânia, cujos membros apresentam bócio multinodular, além de carcinoma papilífero da tiróide, principalmente variante folicular (38).

Esses três genes identificados certamente não são responsáveis por todos os casos de CTNMF. A heterogeneidade genética da doença ainda está longe de ser decifrada completamente e ainda são necessários mais estudos para melhor esclarecimento das bases genéticas do CTNMF.

\section{CONCLUSÕES}

O CTNMF é uma entidade já bem reconhecida, responsável por aproximadamente $5 \%$ dos CDT. Estudos sugerem comportamento geralmente mais agressivo que a forma esporádica e por isso o carcinoma deve ser tratado adequada e rapidamente. Tem sido recomendado, também pela maior agressividade, rastreamento para detecção de CDT em familiares dos pacientes afetados.

Os estudos genéticos ainda são iniciais, mas a identificação completa dos genes responsáveis pela doença pode prover informações essenciais para prevenção, diagnóstico e tratamento, no futuro.

\section{REFERÊNCIAS}

1. Hemminki K, Charis E, Chen B. Familial risks for nonmedullary thyroid cancer. J Clin Endocrinol Metab 2005;90:5747-53.

2. Pal T, Vogl FD, Chappuis PO, Tsang R, Brierley J, Renard H, et al. Increased risk for nonmedullary thyroid cancer in the first degree relatives of prevalent cases of nonmedullary thyroid cancer: a hospital-based study. J Clin Endocrinol Metab 2001;86:5307-12

3. Goldgar DE, Easton DF, Cannon-Albright LA, Skolnick MH. Systematic population-based assessment of cancer risk in first-degree relatives of cancer probands. J Natl Cancer Inst 1994;86:1600-8.

4. Robinson DW, Orr TG. Carcinoma of the thyroid and other diseases of the thyroid in identical twins. AMA Arch Surg 1955;70:923-8.

5. Sturgeon $\mathrm{C}, \mathrm{Clarck} \mathrm{OH}$. Familial nonmedullary thyroid cancer. Thyroid 2005;15:588-93.

6. Alsanea O, Clark OH. Familial thyroid cancer. Curr Opin Oncol 2001;13:44-51.

7. Charkes ND. On the prevalence of familial nommedullary thyroid cancer in multiply affected kindreds. Thyroid 2006;16:181-6.

8. Lupoli G, Vitale G, Caraglia M, Fittipaldi MR, Abbruzzese A, Tagliaferri $P$, et al. Familial papillary thyroid microcarcinoma: a new clinical entity. Lancet 1999;353:637-9.

9. Loh KC. Familial nommedullary thyroid carcinoma: a metareview of case series. Thyroid 1997;7:107-13.

10. Uchino S, Noguchi S, Kawamoto H, Yamashita H, Watanabe S, Shuto S. Familial nonmedullary thyroid carcinoma characterized by multifocality and a high recurrence rate in a large study population. Word J Surg 2002;26:897-902.

11. Musholt TJ, Musholt PB, Petrich T, Oetting G, Knapp WH, Klempnauer J. Familial papillary thyroid carcinoma: genetics, criteria for diagnosis, clinical features and surgical treatment. World J Surg 2000;24:1409-17.

12. Grossman RF, Tu SH, Duh QY, Siperstein AE, Novosolov F, Clark $\mathrm{OH}$. Familial nonmedullary thyroid cancer: an emerging entity that warrants aggressive treatment. Arch Surg 1995; 130:892-7.

13. Maxwell EL, Hall FT, Freeman JL. Familial non-medullary thyroid cancer: a matched-case control study. The Laryngoscope 2004; 114:2182-6.

14. Triponez F, Wong M, Sturgeon C, Caron N, Ginzinger DG, Segal MR, et al. Does familial non-medullary thyroid cancer adversely affect survival? World J Surg 2006;30:787-93.

15. Alsanea O. Familial nommedullary thyroid cancer. Curr Treat Options Oncol 2000;1:345-51.

16. Malchoff CD, Malchoff DM. Familial nonmedullary thyroid carcinoma. Cancer Control 2006;13:106-10. 
17. Vriens MR, Sabanci U, Epstein HD, Ngai S, Duh OY, Siperstein $A E$, et al. Reliability of fine-needle aspiration in patients with familial nonmedullary thyroid cancer. Thyroid 1999;9:1011-16.

18. Uchino S, Noguchi S, Yamashita H, Murakami T, Watanabe S, Ogawa $\mathrm{T}$, et al. Detection of asymptomatic differentiated thyroid carcinoma by neck ultrasonographic screening for familial nonmedullary thyroid carcinoma. World J Surg 2004;28:1099-102.

19. Goldga DE, Easton DF, Cannon-Albright LA, Skolnik MH. Systematic population-based assessment of cancer risk in first degree relatives of cancer probands. J Natl Cancer Inst 1994:86:1600-7.

20. Frich L, Glattre E, Akslen LA. Familial occurrence of nonmedullary thyroid cancer: a population-based study of 5,673 first-degree relatives of thyroid cancer patients from Norway. Cancer Epidemiol Biomarkers Prev 2001; 10:113-7.

21. Lee S, Hong SW, Shin SJ, Kim YM, Rhee Y, Jeon Bl, et al. Papillary thyroid carcinoma associated with familial adenomatous polyposis: molecular analysis of pathogenesis and review of the literature. Endocr J 2004;51(3):317-23.

22. Kovich O, Cohen D. Cowden's syndrome. Dermatol Online J 2004;10:3.

23. Bertherat J. Carney complex. Orphanet J Rare Dis 2006;1:21-6.

24. Randolph GW, Maniar D. Medullary carcinoma of the thyroid. Cancer Control 2000;7:253-9.

25. Burgess JR, Duffield A, Wilkinson J, Ware R, Greenaway TM, Percival J, et al. Two families with an autossomal dominant inheritance pattern for papillary carcinoma of the thyroid. $\mathbf{J}$ Clin Endocrinol Metab 1997;82:345-8.

26. Ron E, Kleinerman RA, LiVolsi VA, Fraumeni JF. Familial nonmedullary thyroid cancer. Oncology 1991:48:309-11.

27. Marchesi M, Biffoni M, Biancari F, Faloci C, Cresti R, Mariotti $\mathrm{R}$, et al. Familial papillary carcinoma of the thyroid: a report of nine first-degree relatives of four families. Eur J Surg Oncol 2000:26:789-91.

28. Orsenigo E, Beretta E, Gini P, Verrechia F, Invernizzi L, Fiorina $P$, et al. A report of six cases of familial papillary thyroid cancer. EJSO 2003:29:185-7.

29. Ríos A, Rodríguez JM, Illana J, Torregrosa NM, Parrila P. Familial papillary carcinoma of the thyroid: report of three families. Eur J Surg 2001;167:339-43.
30. Lote K, Andersen K, Nordal E, Brennhovd O. Familial occurrence of papillary thyroid carcinoma. Cancer 1980;46:1291-7.

31. Leprat F, Bonichont F, Guyot M, Trouette H, Trojani M, Vergnot $V$, et al. Familial non-medullary thyroid carcinomas: pathology review in 27 affected cases from 13 French families. Clin Endocrinol 1999;50:589-94.

32. Ozaki O, Ito K, Kobayashi K, Suzuki A, Manabe Y, Hosoda Y. Familial occurrence of differentiated, non-medullary thyroid carcinoma. World J Surg 1988;12:565-71.

33. Xing M. The T1799A BRAF mutation is not a germline mutation in familial non-medullary thyroid cancer. Clin Endocrinol 2005;63:263-6.

34. Canzian F, Amati P, Harach HR. A gene predisposing to familial thyroid tumors with cell oxiphilia maps to chromossome 19p13.2. Am J Hum Genet 1998;63:1743-8.

35. Bevan S, Pal T, Greenberg CR, Kraimpes JL, Lesueuer F, Barbier J, et al. A comprehensive analysis of MNG1, TCO1, FPTC, PTEN, TSHR and TRK in familial non-medullary thyroid cancer: confirmation of linkage to TCO1. J Clin Endocrinol Metab 2001;86:3701-4

36. McKay JD, Williamson J, Leseur F, Stark M, Duffield S, Canzian $F$, et al. At least three genes account for familial papillary thyroid carcinoma: TCO and MNG1 excluded as susceptibility loci from a large Tasmanian family. Eur J Endocrinol 1999; $141: 122-5$.

37. Malchoff $C D$, Sarfarazi M, Tendler B, Forouhar F. Papillary thyroid carcinoma associated with papillary renal neoplasia: genetic linkage analysis of a distinct heritable tumor syndrome. J Clin Endocrinol Metab 2000;85:1758-64.

38. McKay JD, Lesueur F, Jonard L, Pastore A, Willianson J, Hoffman $L$, et al. Localization of a susceptibility gene for familial non-medullary thyroid carcinoma to chromosome 2q21. Am J Hum Genet 2001;69:440-6.

Endereço para correspondência:

Roberto B. Santos

Rua Dr. Clemente Ferreira 44

13020-120 Campinas, SP

E-mail: robmirsantos@uol.com.br 